\title{
Approach of minimal invasive monitoring and initial treatment of the septic patient in emergency medicine
}

This article was published in the following Dove Press journal:

Open Access Emergency Medicine

\author{
German Devia Jaramillo' \\ Jenny Castro Canoa' \\ Emiro Valverde Galván² \\ 'Emergency Medicine Department, \\ Hospital Universitario Mayor Méderi \\ Universidad del Rosario, Bogotá, \\ Colombia; ${ }^{2}$ Emergency Medicine, \\ Universidad del Rosario, Bogotá, \\ Colombia
}

\begin{abstract}
Sepsis and septic shock constitute a complex disease condition that requires the engagement of several medical specialties. A great number of patients with this disease are constantly admitted to the emergency department, which warrants the need for emergency physicians to lead in the recognition and early management of septic patients. Timely and appropriate interventions may help reduce mortality in a disease with an unacceptably high mortality rate. Poor control of cellular hypoperfusion is one of the most influential mechanisms contributing to the high mortality rate in these patients. This article aims to make an evidence-based approach and an algorithm for the active identification of hypoperfusion in patients with suspicion of severe infection, based on both clinical variables (capillary refill, mottling index, left ventricular function by ultrasound, temperature gradient, etc.) and laboratory-measured variables (lactate, central venous oxygen saturation $\left[\mathrm{ScvO}_{2}\right]$, and venous-to-arterial carbon dioxide tension difference $\left.\left[\mathrm{P}(\mathrm{v}-\mathrm{a}) \mathrm{CO}_{2}\right]\right)$. Such variables are feasible to use in the emergency department and would help to explain the cause behind the inadequate oxygen use by cells, thereby guiding treatment at the macrovascular, microvascular, or cellular level.
\end{abstract} Keywords: sepsis, septic shock, cellular perfusion, microcirculation, emergency medicine

\section{Introduction}

Sepsis is considered a medical emergency. In this sense, the emergency department is responsible for not only identifying this condition but also for appropriately treating patients with the disease. Despite multiple clinical trials and scientific advances in the treatment of sepsis, the mortality rate of this disease continues to be excessively high. In some trials, it ranges between $18 \%{ }^{1,2}$ and $55.7 \% .^{3}$ This mortality difference can be explained by our limited understanding of the disease and the specific and appropriate therapies that are available.

The therapeutic management of septic patients rests on three fundamental pillars. The first pillar involves the medical and/or surgical control of the triggering infection; the second one encompasses supportive therapy for the patient; and the third one is the immunomodulation therapy. Effective intervention in each of these pillars would be expected to reduce the mortality rate. Septic patients may present to the emergency department at various stages of the disease. Some may appear more ill than others; however, they all share the same problem, which is triggered by a variable response to the infectious disease: cellular hypoperfusion. If the patient with sepsis-induced cellular hypoperfusion is not promptly recognized, the outcome will most likely be negative.

The purpose of this review is to propose a practical approach to identify the septic patient with cellular hypoperfusion in the emergency department, and thereby to be able to initiate an early intervention to reduce the mortality rate of this condition.
Correspondence: German Devia Jaramillo Emergency Medicine Department, Hospital Universitario Mayor Méderi Universidad del Rosario, Calle 24-29. Bogota, I I I I I, Colombia, Bogotá, Colombia

Tel/fax +57 I 5600520 ext 396 |

Email german.devia@urosario.edu.co 


\section{Defining hypoperfusion}

In the early stages of sepsis, the inflammatory response to the infection produces multiple cytokines that cause the microcirculation to deteriorate. ${ }^{4}$ The microcirculatory dysfunction may alter the circulatory flow distribution and even stop it, as has been observed in various biological models. ${ }^{5}$ This microcirculatory dysfunction may affect oxygen perfusion to the cell, and its persistence is associated with increased mortality. ${ }^{6,7}$ The patient with microcirculation disturbance is not able to achieve an oxygen demand-supply balance, leading to inadequate oxygen use by the cell - a condition known as cellular hypoperfusion. Despite the mechanisms of compensation, such as anaerobic energy production, hypoperfusion may induce cell, tissue, and organ failure that will trigger death. In the emergency department, the diagnostic challenge revolves around prompt identification of the patient with acute microcirculatory compromise before it becomes irreversible.

\section{Developing an algorithm}

In the emergency department, the physician must be meticulous, methodical, and quick at the same time, given the busy nature of the overcrowded service. Consequently, when confronted with a septic patient, we suggest the use of an algorithm that will allow for a prompt and quick evaluation of the perfusion state on different levels, examining variables from "highest to lowest" in terms of macrocirculation, microcirculation, and the cell.

\section{Macrovascular aspects}

Macrovascular variables refer to the visible or clinically measurable aspects, such as capillary refill, temperature, skin mottling, left ventricular function, mental state, heart rate, blood pressure, and urinary output (Table 1).
Through the three known "windows" of the human body, perfusion may be assessed ${ }^{8}$ (kidney "window": urinary output; brain "window": consciousness; and skin "window": capillary flow), and advanced hypoperfusion states may be detected when physiological compensatory mechanisms are being overwhelmed; nevertheless, in early states, the applicability of these "windows" is less clear. For instance, skin mottling may reflect a state of peripheral vasoconstriction -a clinical manifestation of hypoperfusion. ${ }^{9}$ Based on this, a "mottling score" ranging from 0 to 5 points has been introduced (Table 2). ${ }^{10}$ In a setting of only 60 patients, it showed an association with high mortality within 14 days in septic patients. Similarly, patients whose mottling score decreased following initial resuscitation showed an improved prognosis of death within 14 days - from $77 \%$ to $12 \%{ }^{10}$

Capillary refill is the time taken for the color to return to an external capillary bed after pressure is applied to cause blanching. In a case series of 50 patients, the capillary refill time (CRT) was measured on the fingertip and the knee area, proving to be significantly longer in the patient group that experienced death because of septic shock. It was even observed that a knee CRT $>4.9$ seconds was a predictor of 14-day mortality with a sensitivity of $82 \%(95 \%$ CI $90-95 \%)$ and a specificity of $84 \%$ (95\% CI 68-94\%). ${ }^{11}$

In another study involving patients with sepsis and hyperlactatemia, scientists found that a prolonged capillary refill was associated with an increased risk of adverse outcomes ( $88 \%$ vs $20 \%$; RR 4.4 [2.7-7.4]) and in-hospital mortality (63\% vs 9\%; RR 6.7 [2.9-16]). Moreover, the study revealed that CRT-normal patients required less mechanical ventilation, renal replacement therapy, and admission to the intensive care unit. ${ }^{12}$

Temperature may serve as another marker of cellular perfusion, because patients with lower skin temperature show

Table I Macrovascular aspects for evaluating perfusion in septic patients in the emergency department

\begin{tabular}{|l|l|l|}
\hline Variable & Advantages & Disadvantages \\
\hline Capillary refill & Easy to do, no equipment required & May occur late in presentation, inter-observer variability \\
\hline Temperature & $\begin{array}{l}\text { Does not require complex equipment. Temperature } \\
\text { gradient is preferable over peripheral temperature }\end{array}$ & $\begin{array}{l}\text { May occur late in presentation, not associated with low } \\
\text { organ pulsatility index }\end{array}$ \\
\hline Mottling score & Easy to do, no equipment required & $\begin{array}{l}\text { Inter-observer variability; cannot be used in burn } \\
\text { patients or amputees; and may occur late in presentation }\end{array}$ \\
\hline Urinary output & Easy to do, no complex equipment required & $\begin{array}{l}\text { False positives (urinary obstruction), and false negatives } \\
\text { (diuretic therapy) may occur }\end{array}$ \\
\hline Mental state & Easy to do, no equipment required & $\begin{array}{l}\text { May occur late in presentation. False positives are } \\
\text { common (stroke, metabolic encephalopathy, electrolytic } \\
\text { disturbance) }\end{array}$ \\
\hline Left ventricular strain & $\begin{array}{l}\text { Direct evaluation of cardiac contraction, may be } \\
\text { undertaken at the bedside }\end{array}$ & $\begin{array}{l}\text { Requires minimal training; has inter-observer variability } \\
\text { and false positives (baseline systolic dysfunction) }\end{array}$ \\
\hline Vital signs (Shock Index) & Easy to do, no complex equipment required & May be a cause or consequence of hypoperfusion \\
\hline
\end{tabular}


Table 2 The mottling score

\begin{tabular}{|l|l|}
\hline Score & Mottling area extension on the legs \\
\hline 0 & No mottling \\
\hline $\mathrm{I}$ & $\begin{array}{l}\text { A modest mottling area (coin size) localized to the center of } \\
\text { the knee }\end{array}$ \\
\hline 2 & $\begin{array}{l}\text { Moderate mottling area that does not exceed the superior } \\
\text { edge of the kneecap }\end{array}$ \\
\hline 3 & Mild mottling area that does not exceed the middle thigh \\
\hline 4 & $\begin{array}{l}\text { Severe mottling area that does not go beyond the fold of } \\
\text { the groin }\end{array}$ \\
\hline 5 & $\begin{array}{l}\text { Extremely severe mottling area that goes beyond the fold of } \\
\text { the groin }\end{array}$ \\
\hline
\end{tabular}

low cardiac index and $\mathrm{ScvO}_{2}$, and higher lactate values than those with higher skin temperatures. ${ }^{13}$ A study including 40 septic patients revealed that, in patients with septic shock, the core-to-toe temperature gradient was significantly higher than in patients with severe sepsis $\left(12.5^{\circ} \mathrm{C}\right.$ [9.2-13.8] vs $6.9^{\circ} \mathrm{C}$ [3.4-12], $\left.P<0.001\right)$. After initial resuscitation, the toe-to-room temperature gradient was lower in patients who died from multiple organic dysfunction than in survivors $\left(-0.2^{\circ} \mathrm{C}[-1.1 ;+1.3]\right.$ vs $\left.+3.9^{\circ} \mathrm{C}[+0.5 ;+7.2], P<0.001\right)$. The toe-to-room temperature gradient was related to the arterial lactate values $(r=0.54, P<0.0001)$, urinary output $(r=0.37$, $P=0.0002)$, knee capillary refill $(r=0.42, P<0.0001)$, and mottling score $(P=0.001) .{ }^{14}$

In a prospective pilot study with 30 patients, ${ }^{15}$ scientists sought to establish the relationship among variables of clinical perfusion, such as capillary refill, peripheral temperature, mottling score, and pulsatile indexes (measured by Doppler ultrasound) of the intestines, liver, kidney, and spleen. A statistically significant relationship was found between the pulsatile index of the organs and the CRT (intestine: $r=0.325, P=0.007$ ) as well as the mottling score (kidney: $r=0.396, P=0.006$ ) albeit not with the peripheral temperature.

The percentage distance shortening of the endocardium along its length is known as the left ventricular strain, and it is a measure of cardiac ejection fraction. Left ventricular strain may provide information about myocardial function and detect cardiac disturbances early as the cause or consequence of cellular hypoperfusion. ${ }^{9}$ In a recent study involving 89 patients, scientists found that patients reporting severe abnormalities in the left ventricular longitudinal strain had a lower $\mathrm{ScvO}_{2}(56.1 \%$ vs $67.5 \%, P<0.01)$ and higher lactate level $(2.7$ vs $1.9 \mathrm{mmol} / \mathrm{dL}, P=0.04) .{ }^{16}$ In the emergency department, it is possible to undertake these calculations; however, ultrasound training is required and, because the measurement is dependent on the observer's experience, inaccurate results may be obtained.
Some vital signs, such as heart rate, blood pressure, and respiratory rate, may yield abnormal values as a manifestation of cellular hypoperfusion, showing a physiological compensatory mechanism. However, these abnormal values may result from the derangement leading to this hypoperfusion. Such is the case, for example, when a patient with hypoperfusion has tachycardia. The high rate may be a compensatory mechanism by which the heart tries to increase its cardiac output, or it may be limiting cardiac refill and causing hypoperfusion.

Similarly, low blood pressure can be the cause of cellular hypoperfusion in patients with low cardiac output and a decreased microvascular flow; however, it can result from cellular dysfunction of the cardiovascular system.

Emergency physicians should know how to interpret vital signs in critically ill patients because abnormalities may occur not only because of hypoperfusion, but also as a result of damaged microcirculation.

In a retrospective study involving 50 patients, it was found that the Shock Index (a measurement of the relationship between the heart rate and the systolic blood pressure) taken after 2 hours of admission to the emergency department was associated with an in-hospital mortality rate - having a sensitivity of $80.8 \%$, specificity of $79.2 \%$, and AUC value of $0.8894(95 \%$ CI $0.8052-0.9736)$ at a cutoff point greater than or equal to $1.0 .^{17}$

\section{Microvascular aspects}

The microcirculation comprises blood vessels of less than $100 \mu \mathrm{m}$ (arterioles, capillaries, and venules). ${ }^{5}$ The maximum tissue oxygen delivery occurs in this microvascular space, in which various specialized cells - endothelial, smooth muscle, red and white blood cells, and platelets - may be found. Among these, endothelial cells play a key role in regulating microcirculation, because they mediate thrombosis and fibrinolysis, leukocyte adhesion and migration, vasomotor tone, exchange of nutrients and other cells, and capillary permeability and recruitment.

Under normal physiological conditions, nitric oxide plays a crucial role in distributing the microvascular flow to meet the metabolic needs of tissues. Failure in nitric oxide mechanisms constitutes an important cause of perfusion alterations in septic patients. ${ }^{18}$

Other endothelial cell alterations in septic patients include glycocalyx degradation and deformability of blood cells. ${ }^{5}$ These alterations, among others, are the cause of cellular hypoperfusion - that is, inadequate oxygen use leading to organic dysfunction and death. 
In order to promptly identify patients with hypoperfusion before macrocirculation alterations become clinically evident, some variables can be measured, including arterial, capillary, or venous lactate levels, $\mathrm{ScvO}_{2}$, venous-to-arterial carbon dioxide tension difference $\left(\mathrm{P}[\mathrm{v}-\mathrm{a}] \mathrm{CO}_{2}\right)$, tissue oxygen saturation, and near-infrared spectroscopy (NIRS). In the emergency department, there is a need for readily reproducible variables that do not require complex measurement equipment (Table 3).

Lactate is the product of anaerobic glycolysis. It increases in states of cellular hypoxia and is, therefore, used as a perfusion marker. ${ }^{19}$ However, lactate elevation occurs in accelerated aerobic glycolysis as a stress response. ${ }^{20,21}$ Thus, high lactate levels may originate both from a state of hypoxia/ hypoperfusion and aerobic glycolysis, making laboratory results difficult to interpret for the emergency physician. Increased lactate values are not always due to cellular hypoxia/hypoperfusion, which is why interventions pursuing lactate clearance based on the improvement of oxygen delivery could be harmful in cases of high lactate levels secondary to an accelerated aerobic glycolysis. ${ }^{22}$

Lactate measurement is useful in the resuscitation of septic patients in the emergency department. In a study conducted in a single hospital, median lactate values of 2.9 $\mathrm{mmol} / \mathrm{L}$ had a 28 -day mortality rate of $22.9 \%$. Intermediate and high values of lactate were associated with a high mortality rate in non-shock patients $(\mathrm{OR}=2.05, P=0.025$, and $\mathrm{OR}=4.87, P<0.001$, respectively) and an even higher rate in the shock group (intermediate values: $\mathrm{OR}=3.2, P=0.022$; high values $\mathrm{OR}=4.8, P=0.001) .{ }^{23}$

Furthermore, the clearance of lactate values may help to predict the prognosis of patients with sepsis. In a study with 111 patients conducted in the emergency department, there was an $11 \%$ decreased likelihood of mortality for every $10 \%$ in lactate clearance. Patients with a lactate clearance $>10 \%$ showed a greater decrease in the APACHE II score at 72 hours and lower 60-day mortality $(P=0.007) .{ }^{24}$ Lactic acidosis may be produced by multiple processes; nevertheless, it is essential to establish whether the acidosis has a hypoxic origin. The relationship between arterial lactate and pyruvate levels may be considered in this identification, as a $>10 \%$ relationship is indicative of tissue hypoxia. ${ }^{25}$

The aforementioned studies used arterial lactate values; however, a recent study involving 79 patients revealed an area under the curve for capillary lactate of $82 \%$ (95\% CI 73-91\%). The best cutoff point was $2.35 \mathrm{mmol} / \mathrm{L}$, with a sensitivity of $81 \%(95 \%$ CI $65-90 \%)$ and a specificity of $70 \%(95 \%$ CI $53-83 \%) .{ }^{26}$ This could be factored in to argue in favor of the use of capillary lactate values in the future for predicting and guiding the resuscitation of septic patients in the emergency department.

The $\mathrm{ScvO}_{2}$ is a marker of adequate oxygen delivery, ${ }^{9}$ and a low $\mathrm{ScvO}_{2}$ value may reflect an abnormal oxygen delivery $\left(\mathrm{DO}_{2}\right),{ }^{27}$ owing to a greater oxygen extraction ratio from increased oxygen consumption as a compensatory mechanism. ${ }^{28}$ In a study including 619 septic patients, $\mathrm{ScvO}_{2}$ values were stratified in three levels: hypoxia $(<70 \%)$, normoxia (71-89\%), and hyperoxia (90-100\%). It was found that the mortality rate was statistically lower in the normoxia group $(21 \%, 17-25 \%)$ as compared with the hyperoxia $(40 \%$, $29-53 \%)$ and hypoxia groups $(40 \%, 29-53 \%)$. In the multivariate analysis, the hyperoxia group also had the highest association with mortality (OR.2.2; 95\% CI 1.3-3.7). ${ }^{29}$

Both lactate clearance and $\mathrm{ScvO}_{2}$ improvement may be used in the resuscitation of septic patients in the emergency department. In a study involving 300 patients admitted to the emergency department with a diagnosis of sepsis, these two parameters were compared. In one group, resuscitation was guided until lactate clearance and, in the second group, resuscitation was guided until normalization of the $\mathrm{ScvO}_{2}$. Scientists found no difference in the treatment administered during the first 72 hours of hospitalization. A

Table 3 Microvascular aspects for evaluating perfusion in septic patients in the emergency department

\begin{tabular}{|c|c|c|}
\hline Variable & Advantages & Disadvantages \\
\hline Lactate & $\begin{array}{l}\text { In terms of mortality, clearance is associated with lower } \\
\text { mortality, it is easy to perform, and can be sampled } \\
\text { from the capillary. In the appropriate context, it may } \\
\text { reflect hypoxia/hypoperfusion. }\end{array}$ & $\begin{array}{l}\text { Elevated values are not necessarily due to } \\
\text { hypoperfusion, as they may result from } \\
\text { increased aerobic glycolysis or a metabolic } \\
\text { response to stress. } \\
\text { Requires a machine. }\end{array}$ \\
\hline $\mathrm{ScvO}_{2}$ & $\begin{array}{l}\text { A low value may indicate low oxygen delivery, or even } \\
\text { an increase in the extraction ratio. It may be prognostic. }\end{array}$ & $\begin{array}{l}\text { Requires a central venous line. It is not } \\
\text { better than lactate as a resuscitation guide. }\end{array}$ \\
\hline $\mathrm{PCO}_{2}$ gap & $\begin{array}{l}\text { It may indicate microcirculatory disturbance, mainly due } \\
\text { to cardiac output alteration. It may be prognostic. }\end{array}$ & Requires a central venous line. \\
\hline $\begin{array}{l}\mathrm{PtiO}_{2}, \mathrm{NIRS} \text {, video } \\
\text { microscopy }\end{array}$ & $\begin{array}{l}\text { Allows for a real-time approach to microcirculation. It } \\
\text { may be prognostic. }\end{array}$ & $\begin{array}{l}\text { Requires complex equipment. } \\
\text { Its clinical value has yet to be established. }\end{array}$ \\
\hline
\end{tabular}


total of $23 \%$ of patients who were resuscitated to the $\mathrm{ScvO}_{2}$ normalization died (95\% CI 17-30\%), and 17\% of patients resuscitated to lactate clearance died (95\% CI 11-24\%). This difference did not reach statistical significance, and there was no difference in treatment-related adverse events between both groups. ${ }^{30}$

The venous-to-arterial carbon dioxide tension difference $\left(\triangle \mathrm{PCO}_{2}\right)$ is the difference between the partial pressure of $\mathrm{CO}_{2}$ in mixed venous blood or central venous blood $\left(\mathrm{PvCO}_{2}\right.$ or $\mathrm{PcvCO}_{2}$ ) and the partial pressure of $\mathrm{CO}_{2}$ in arterial blood $\left(\mathrm{PaCO}_{2}\right)$ :

$$
\triangle \mathrm{PCO}_{2}=\mathrm{PcvCO}_{2}-\mathrm{PaCO}_{2} \text {. (normal values } 2-5 \mathrm{mmHg} \text { ) }
$$

$\triangle \mathrm{PCO} 2$ is considered a marker of the venous microvascular flow capacity to eliminate $\mathrm{CO}_{2}$ from the peripheral tissue, ${ }^{9}$ and biological models have confirmed these results. ${ }^{31}$ Consequently, this measurement is not only an indirect marker of cellular hypoxia but also a marker of venous flow and cardiac output. Under normal physiological conditions, the standard value must be between 2 and $5 \mathrm{mmHg}$. An increase may indicate insufficient cardiac output to supply the metabolic needs of the body. ${ }^{31}$

In a study with 75 septic patients, the $\mathrm{PCO}_{2}$ gap was compared through video microscopic techniques for evaluating microcirculation, and a significant correlation was found between a high $\mathrm{PCO}_{2}$ gap and a reduced percentage of perfused blood vessels. ${ }^{32}$ We can, therefore, suggest the use of the $\mathrm{PCO}_{2}$ gap as a marker of microvascular flow dysfunction because of cardiac output abnormality.

In a retrospective study with 172 septic patients, the $\mathrm{ScvO}_{2}$ and the $\mathrm{PCO}_{2}$ gap were combined to evaluate the prognosis and mortality of patients. The 28-day mortality was $35 \%$ in the overall population, and for patients in whom the $\mathrm{SvcO}_{2}$ could not be improved at 6 hours, the mortality rate was $50 \%$, as compared to a mortality rate of $29.5 \%$ in patients with an $\mathrm{ScvO}_{2}>70 \%(P=0.009)$. In the patient group with $\mathrm{ScvO}_{2}>70 \%$, mortality was even lower if the $\mathrm{PCO}_{2}$ gap was $<6 \mathrm{mmHg}$, compared to a $\mathrm{PCO}_{2}$ gap $>6(56.1 \%$ vs $16.1 \% ; P=0.001) .{ }^{33}$ The $\mathrm{ScvO}_{2}$ and the $\mathrm{PCO}_{2}$ gap may be used together not only as a marker of hypoperfusion, but also as a prognostic tool in the emergency department.

Other techniques, such as microcirculation evaluation with video microscopy or even NIRS, could be useful for determining cellular perfusion, if available in the emergency department. In a recent review, MacDonald et al proposed the use of NIRS in the emergency department because of the portable technology of the device; however, its clinical value is yet to be established..$^{34}$

\section{Other biomarkers}

\section{Procalcitonin (PCT)}

PCT is a calcitonin propeptide that is normally produced by thyroid, gastrointestinal, and pulmonary tissue. Bacterial infections, through inflammatory mediators, cause their production to increase and, in viral infections, their production is inhibited by the increase of interferon- $\gamma$. Levels begin to increase within 3-6 hours and peak at 6-22 hours with bacterial infection. With infection resolution, levels typically decrease by $50 \%$ per day. ${ }^{35}$ Thanks to these characteristics, PCT could be used with caution to define the continuity of antibiotic therapy or even for the diagnosis of bacterial sepsis. It is not a direct index of perfusion; however, it could be used as a prognostic marker of mortality; a study compared it with other markers such as lactate and prognostic scores and found that values $>15.05 \mathrm{ng} / \mathrm{mL}$ are predictors of mortality at 28 days (AUC 0.92). ${ }^{36}$

\section{Troponin}

Troponin is the biomarker of choice for the diagnosis of myocardial infarction; however, it may be elevated in 36-85\% of patients with sepsis ${ }^{37}$ and could be used as an indicator of associated cardiac injury. Its detection in blood is related to increased mortality ${ }^{38}$ and could be used as a marker of cardiac hypoperfusion.

\section{Mid-regional proadrenomedullin}

Adrenomedullin (ADM) is a peptide that is produced by the adrenal medulla. ADM is produced during physiological stress such as sepsis and has various functions including vasodilation and anti-inflammatory and antimicrobial effects; ${ }^{39}$ the circulating mid-regional proADM levels are significantly higher in patients with sepsis than in patients with systemic inflammatory response syndrome (SIRS); therefore, given that ADM is rapidly cleared from circulation, the levels of proadrenomedullin (proADM) are used as a surrogate. ADM is responsible for hypotension associated with severe sepsis, which has been proposed as a good marker for risk assessment and predicting sepsis prognosis. ${ }^{40}$

\section{Angiopoietin}

Angiopoietin (Ang) 1 and 2 are endothelial-derived vascular growth factors that play opposing roles during sepsis. Ang-1 stabilizes the endothelium, whereas Ang-2 facilitates loss of endothelial integrity and vascular leakage. ${ }^{41}$ Elevated levels of circulating Ang-2 are associated with sepsis with multiorgan dysfunction, which is indicative of impaired vascular endothelial integrity. ${ }^{41}$ 
Direct biomarkers of endothelial damage could be of great interest during sepsis. For instance, adhesion molecules (intercellular adhesion molecule-1, vascular cell adhesion molecule-1, E-selectin, and P-selectin) can be assayed in the serum of septic patients and reflect endothelial cell activation (or endothelium disruption) and increased leukocyte-endothelial interactions. ${ }^{42}$

\section{Cellular aspects}

Mitochondrial dysfunction becomes a key element in the pathology and recovery of the septic process. When there is mitochondrial dysfunction, micro- and macrocirculatory improvement does not correlate with the improvement of optimal cellular perfusion. ${ }^{43}$ Thus, evaluating the mitochondrial respiration should be an unquestionable aim. At the moment of this review, there are various methods for measuring mitochondrial oxygenation; ${ }^{5,44}$ nevertheless, these methods are hard to apply at the emergency department, and there is a need for noninvasive, easy-to-use techniques for these oxygenation values.

A recent study in the setting of an endotoxin-induced septic animal model evaluated the monitoring of mitochondrial oxygen pressure $\left(\mathrm{MitoPO}_{2}\right)$ and local mitochondrial use of oxygen by means of a protoporphyrin IX-triplet state lifetime technique (PplX-TSLT), which yielded promising results. ${ }^{45}$ This is an initial contribution that pursues the monitoring of mitochondrial function non-invasively and one that can be performed at the bedside. Improvements in mitochondrial respiration, function, and movement should be a research target in the short term to potentially decrease the high mortality rates of septic patients.

In brief, we propose an algorithm for identifying patients with hypoperfusion in the emergency department, involving the previously described macrovascular, microvascular, and cellular aspects (Figure 1).

\section{Using the algorithm}

Sepsis is defined as a life-threatening organ dysfunction caused by a dysregulated host response to infection. ${ }^{46}$ and septic shock is defined as a subset of sepsis in which the underlying circulatory and cellular metabolism abnormalities are profound enough to substantially increase mortality. ${ }^{46}$ Interestingly, at present, emphasis is placed on organic failure as the cause of mortality due to sepsis. This organ dysfunction is secondary to the damage to the cellular perfusion secondary to the injury of the microcirculation that is caused by the inflammatory process triggered by the infection. ${ }^{5}$ The hypoperfusion detection algorithm proposed in this article can be applied to the admission of the patient with clinical suspicion of infection to the emergency room - that is, it can be used as a complement to indices of organic dysfunction such as quickSOFA (qSOFA) or Sequential Organ Failure

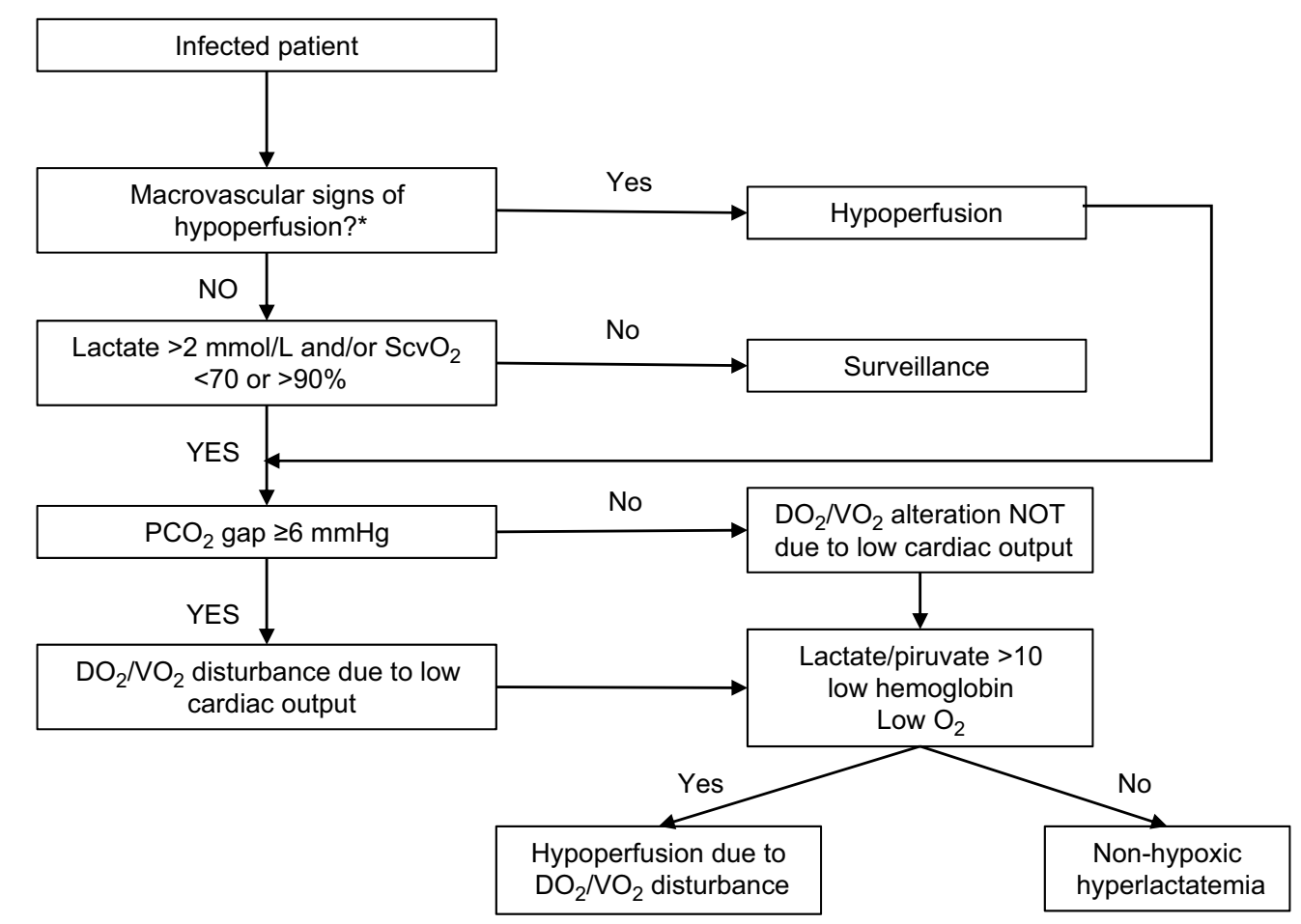

Figure I Algorithm for identifying hypoperfusion in septic patients in the emergency department. Note: *Capillary refill, mottling score, temperature, urinary output, mental state, ventricular strain, and vital signs. 
Assessment (SOFA) in the evaluation of the patient with suspected sepsis in the emergency department.

\section{Treating hypoperfusion}

One of the fundamental pillars in the treatment of septic patients is supportive therapy - that is, interventions established to relieve the different cellular and tissue abnormalities that occur as the infectious process is controlled. There are published and specific recommendations for treating patients with septic shock; ${ }^{47}$ nonetheless, there are other strategies based on the improvement of perfusion markers. A study involving 30 septic patients compared the "standard" therapy against clinical perfusion indices and tissue oxygen saturation therapy, revealing that this last group required less resuscitation fluid (statistically non-significant, $P=0.39$ ), and had fewer days of hospital stay (16 vs 43 days, $P=0.05$ ) as well as lower organ dysfunction scores (SOFA) ${ }^{48}$ However, more studies are needed to recommend this treatment protocol.

Furthermore, we suggest an algorithm for treating septic patients based on the cause of hypoperfusion (Figure 2).

The first step is to identify hypoperfusion in the patient with a suspected infection. Next, a systematic search of the cause of hypoperfusion is recommended. A derangement in the $\mathrm{DO}_{2} / \mathrm{VO}_{2}$ relationship should be checked for in patients with suspicion of hypoperfusion. The causes of high lactate values should be addressed based on the right clinical context, as with diminished $\mathrm{ScvO}_{2}$ due to an alteration in $\mathrm{DO}_{2}$. In case of derangement, the next step is to evaluate whether there is low microcirculatory flow (secondary to low cardiac output) by measuring the $\mathrm{PCO}_{2}$ gap. If this gap is elevated, we suggest increasing the cardiac output by means of a fluid challenge guided by the tools for prediction of fluid responsiveness (passive leg raising [PLR], collapsibility index of the inferior vena cava [IVCCI], and pulse pressure variation [PPV]). ${ }^{49}$

If the patient is being mechanically ventilated, he/she should be assessed with one or more of the available tools - PLR, IVCCI, or a PPV. If the patient is not under invasive mechanical ventilation or has an arrhythmia, the method of evaluation for fluid responsiveness should involve PLR.

The PLR test consists of measuring the hemodynamic effects of leg elevation from $0^{\circ}$ to $45^{\circ}$ It is considered positive for a volume response when the change in cardiac output is $>10 \%$ with limb elevation. ${ }^{50}$

The IVCCI comprises of an observation of the variation of the diameter of the inferior vena cava associated with the respiratory movements, following the two-dimensional visualization of the inferior vena cava using the subcostal view of the long axis approximately $2 \mathrm{~cm}$ from the entrance to the right atrium. Indices $>12 \%$ in patients with mechanical ventilation $^{51}$ and $>39 \%$ in patients with spontaneous breathing are predictors of the volume response. ${ }^{52}$

$$
\operatorname{IVCCI}(\%)=100 \times(\mathrm{eIVC}-\mathrm{iIVC}) / \mathrm{eIVC}
$$

PPV consists of evaluating the change in pulse pressure with respiratory movements in a patient under mechanical ventilation; with a tidal volume of $6-8 \mathrm{~mL} / \mathrm{kg}$, a change $>3.5 \%$ is a predictor of response to fluids and, with a tidal

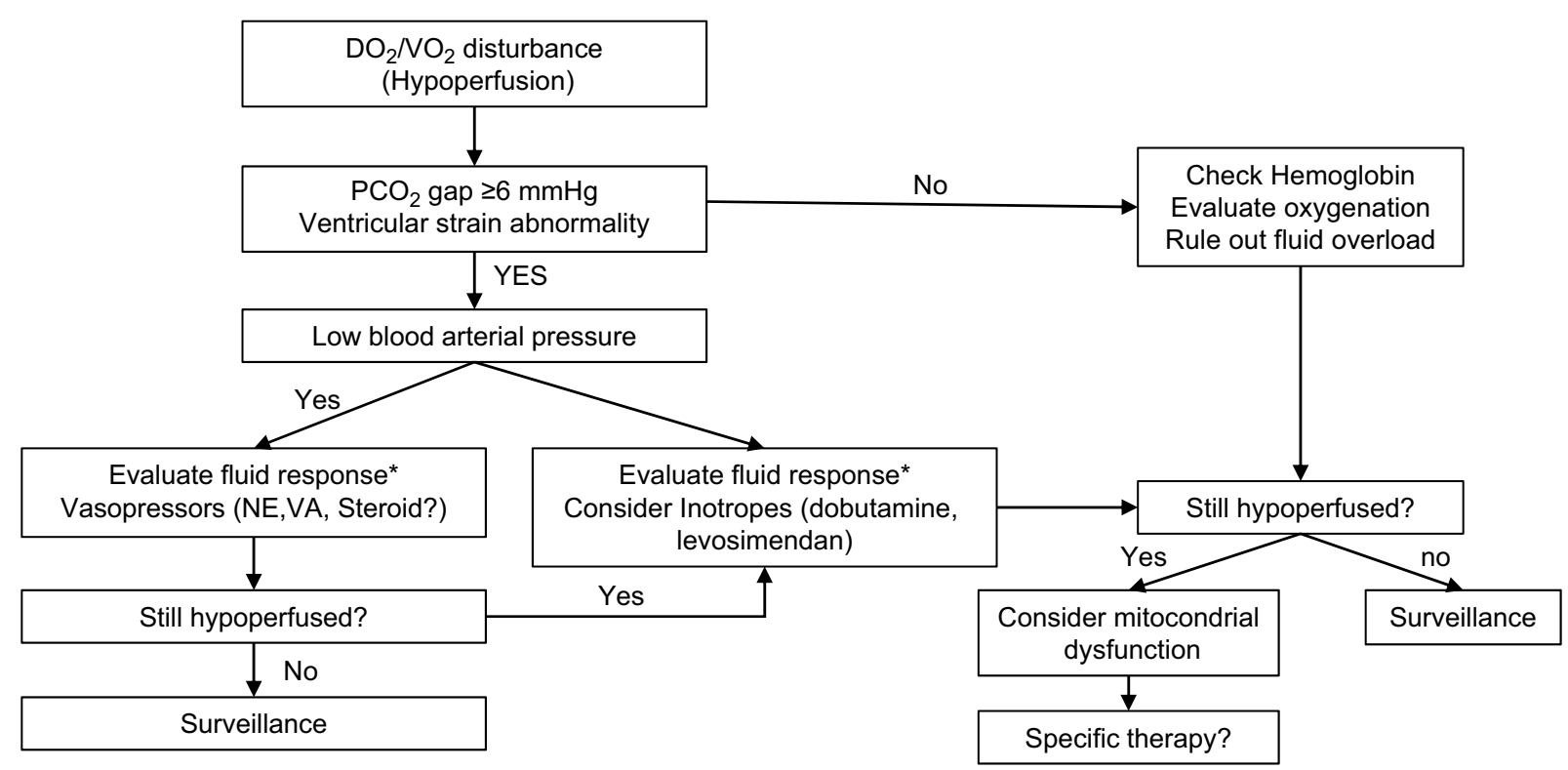

Figure 2 Algorithm for treating hypoperfusion in septic patients in the emergency department.

Notes: *Mechanically ventilated: PLR, IVCCI, and PPV; Spontaneous breathing: PLR, IVCCI.

Abbreviations: IVCCl, collapsibility index of the inferior vena cava; NE, norepinephrine; PLR, passive leg raising; PPV, pulse pressure variation; VA, vasopressin. 
volume of 8 a $12 \mathrm{~mL} / \mathrm{kg}$, a change $>12 \%$ is a predictor of fluid response. ${ }^{51}$

In case the patient does not show signs of being fluid responsive, the next step is to evaluate blood pressure and decide whether the patient requires vasopressors to improve arterial blood pressure. Where a cardiac output abnormality is suspected and blood pressure is normal, the use of an inotrope may be beneficial. This may be further assessed with an ultrasound measurement of ventricular strain. If there is no suspicion of cardiac output alteration, the patient should be evaluated for need of oxygenation with positive pressure or hemoglobin concentration to look for anemia. It is important that the emergency physician be careful not to miss fluid overload and interstitial edema.

The last step to be considered for patients with cellular hypoperfusion is the mitochondrial-cellular dysfunction. Treatment strategies to control mitochondrial dysfunction include improving the use of oxygen, ATP generation, electron flux transport system, oxidative stress, and membrane integrity, as well as a decrease in inflammatory and apoptotic signals. ${ }^{53} \mathrm{~A}$ fundamental aspect of mitochondrial dysfunction is oxidative stress in sepsis, from an imbalance between the production of ROS and antioxidant mechanisms. ${ }^{54}$ Consequently, antioxidant therapy should be advantageous to balance such a mismatch. However, it should involve selective mitochondrial antioxidants, because non-selective therapy has proven to be ineffective. Selective antioxidant therapy may be administered exogenously through compounds or endogenously by promoting endogenous antioxidants. ${ }^{55}$ All these therapies have a long way to go before becoming routine treatment options.

This review is intended to suggest managing sepsisinduced hypoperfusion based on markers within clinical parameters that are feasible to pursue in the emergency department; nevertheless, this is founded on supportive therapy. Interventions based on source control of infection or specific immunomodulation therapies, although interesting, are beyond the scope of this review.

\section{Conclusion}

Emergency physicians should lead the early identification of hypoperfusion in patients with suspicion of infection to begin appropriate interventions and thereby prevent the progression of derangement and subsequent cellular death. To this end, this review recommends the use of clinical and laboratory tools of easy access in the emergency department. Based on perfusion markers, an algorithm has been suggested that involves therapeutic interventions to establish an adequate use of cellular oxygen. The treatment of the septic patient should be tailored individually; it should encompass measures for both infection control and support, and consider immunomodulation therapy, which to date has not been completely established due to the heterogeneity of clinical trials.

\section{Disclosure}

The authors report no conflicts of interest in this work.

\section{References}

1. ARISE Investigators, ANZICS Clinical Trials Group, Peake SL, et al. Goal-directed resuscitation for patients with early septic shock. NEngl J Med. 2014;371(16):1496-1506.

2. ProCESS Investigators, Yealy DM, Kellum JA, et al. A randomized trial of protocol-based care for early septic shock. NEngl J Med. 2014;370(18): $1683-1693$.

3. Machado FR, Cavalcanti AB, Bozza FA, et al. The epidemiology of sepsis in Brazilian intensive care units (the Sepsis PREvalence Assessment Database, SPREAD): an observational study. Lancet Infect Dis. 2017;17(11):1180-1189.

4. Cohen J. The immunopathogenesis of sepsis. Nature. 2002;420(6917): 885-891.

5. Miranda M, Balarini M, Caixeta D, Bouskela E. Microcirculatory dysfunction in sepsis: pathophysiology, clinical monitoring, and potential therapies. Am J Physiol Heart Circ Physiol. 2016;311(1):H24-H35.

6. Trzeciak S, Dellinger RP, Parrillo JE, et al. Early microcirculatory perfusion derangements in patients with severe sepsis and septic shock: relationship to hemodynamics, oxygen transport, and survival. Ann Emerg Med. 2007;49(1):88-98, 98.e1-e2.

7. Sakr Y, Dubois MJ, de Backer D, Creteur J, Vincent JL. Persistent microcirculatory alterations are associated with organ failure and death in patients with septic shock. Crit Care Med. 2004;32(9): 1825-1831.

8. Vincent JL, Ince C, Bakker J. Clinical review: Circulatory shock-an update: a tribute to Professor Max Harry Weil. Crit Care. 2012;16(6):239.

9. Hasanin A, Mukhtar A, Nassar H. Perfusion indices revisited. J Intensive Care. 2017;5:24.

10. Ait-Oufella H, Lemoinne S, Boelle PY, et al. Mottling score predicts survival in septic shock. Intensive Care Med. 2011;37(5):801-807.

11. Ait-Oufella H, Bige N, Boelle PY, et al. Capillary refill time exploration during septic shock. Intensive Care Med. 2014;40(7):958-964.

12. Lara B, Enberg L, Ortega M, et al. Capillary refill time during fluid resuscitation in patients with sepsis-related hyperlactatemia at the emergency department is related to mortality. PLoS One. 2017;12(11): e0188548.

13. Kaplan LJ, McPartland K, Santora TA, Trooskin SZ. Start with a subjective assessment of skin temperature to identify hypoperfusion in intensive care unit patients. J Trauma. 2001;50(4):620-628.

14. Bourcier S, Pichereau C, Boelle PY, et al. Toe-to-room temperature gradient correlates with tissue perfusion and predicts outcome in selected critically ill patients with severe infections. Ann Intensive Care. 2016;6(1):63.

15. Brunauer A, Koköfer A, Bataar O, et al. Changes in peripheral perfusion relate to visceral organ perfusion in early septic shock: A pilot study. $J$ Crit Care. 2016;35:105-109.

16. Lanspa MJ, Pittman JE, Hirshberg EL, et al. Association of left ventricular longitudinal strain with central venous oxygen saturation and serum lactate in patients with early severe sepsis and septic shock. Crit Care. 2015;19:304.

17. Yussof SJ, Zakaria MI, Mohamed FL, Bujang MA, Lakshmanan $\mathrm{S}$, Asaari AH. Value of Shock Index in prognosticating the shortterm outcome of death for patients presenting with severe sepsis and septic shock in the emergency department. Med $J$ Malaysia. 2012;67(4):406-411. 
18. Trzeciak S, Cinel I, Phillip Dellinger R, et al. Resuscitating the microcirculation in sepsis: the central role of nitric oxide, emerging concepts for novel therapies, and challenges for clinical trials. Acad Emerg Med. 2008;15(5):399-413.

19. Fuller BM, Dellinger RP. Lactate as a hemodynamic marker in the critically ill. Curr Opin Crit Care. 2012;18(3):267-272.

20. Garcia-Alvarez M, Marik P, Bellomo R. Sepsis-associated hyperlactatemia. Crit Care. 2014;18(5):503.

21. Kraut JA, Madias NE, Acidosis L. Lactic acidosis. $N$ Engl J Med. 2014;371(24):2309-2319.

22. Kushimoto S, Akaishi S, Sato T, et al. Lactate, a useful marker for disease mortality and severity but an unreliable marker of tissue hypoxia/ hypoperfusion in critically ill patients. Acute Med Surg. 2016;3(4) 293-297.

23. Mikkelsen ME, Miltiades AN, Gaieski DF, et al. Serum lactate is associated with mortality in severe sepsis independent of organ failure and shock. Crit Care Med. 2009;37(5):1670-1677.

24. Nguyen HB, Rivers EP, Knoblich BP, et al. Early lactate clearance is associated with improved outcome in severe sepsis and septic shock Crit Care Med. 2004;32(8):1637-1642.

25. Levy B, Sadoune LO, Gelot AM, Bollaert PE, Nabet P, Larcan A. Evolution of lactate/pyruvate and arterial ketone body ratios in the early course of catecholamine-treated septic shock. Crit Care Med. 2000;28(1):114-119.

26. Seoane L, Papasidero M, de Sanctis P, Posadas-Martínez LM, Soler S, Rodríguez M. Capillary lactic acid validation in an ED. Am J Emerg Med. 2013;31(9):1365-1367.

27. Rosário AL, Park M, Brunialti $\mathrm{MK}$, et al. $\mathrm{SvO}(2)$-guided resuscitation for experimental septic shock: effects of fluid infusion and dobutamine on hemodynamics, inflammatory response, and cardiovascular oxidative stress. Shock. 2011;36(6):604-612.

28. Rivers EP, Yataco AC, Jaehne AK, Gill J, Disselkamp M. Oxygen extraction and perfusion markers in severe sepsis and septic shock: diagnostic, therapeutic and outcome implications. Curr Opin Crit Care. 2015;21(5):381-387.

29. Pope JV, Jones AE, Gaieski DF, et al. Multicenter study of central venous oxygen saturation $(\mathrm{ScvO}(2))$ as a predictor of mortality in patients with sepsis. Ann Emerg Med. 2010;55(1):40-46.e1.

30. Jones AE, Shapiro NI, Trzeciak S, et al. Lactate clearance vs central venous oxygen saturation as goals of early sepsis therapy: a randomized clinical trial. JAMA. 2010;303(8):739-746.

31. Lamia B, Monnet X, Teboul JL. Meaning of arterio-venous $\mathrm{PCO} 2$ difference in circulatory shock. Minerva Anestesiol. 2006;72(6):597-604.

32. Ospina-Tascón GA, Umaña M, Bermúdez WF, et al. Can venous-toarterial carbon dioxide differences reflect microcirculatory alterations in patients with septic shock? Intensive Care Med. 2016;42(2):211-221.

33. Du W, Liu DW, Wang XT, et al. Combining central venous-to-arterial partial pressure of carbon dioxide difference and central venous oxygen saturation to guide resuscitation in septic shock. J Crit Care. 2013;28(6):1110.e1-1110.e5.

34. MacDonald SP, Brown SG. Near-infrared spectroscopy in the assessment of suspected sepsis in the emergency department. Emerg Med J. 2015;32(5):404-408

35. Clerico A, Plebani M. Biomarkers for sepsis: an unfinished journey. Clin Chem Lab Med. 2013;51(6):1135-1138.
36. Mustafić S, Brkić S, Prnjavorac B, Sinanović A, Porobić Jahić H, Salkić S. Diagnostic and prognostic value of procalcitonin in patients with sepsis. Med Glas. 2018;15(2):93-100.

37. Hamilton MA, Toner A, Cecconi M. Troponin in critically ill patients. Minerva Anestesiol. 2012;78(9):1039-1045.

38. Long B, Koyfman A. Ready for Prime Time? Biomarkers in Sepsis. Emerg Med Clin North Am. 2017;35(1):109-122.

39. Linscheid P, Seboek D, Zulewski H, Keller U, Müller B. Autocrine/ paracrine role of inflammation-mediated calcitonin gene-related peptide and adrenomedullin expression in human adipose tissue. Endocrinology. 2005;146(6):2699-2708.

40. Christ-Crain M, Morgenthaler NG, Struck J, Harbarth S, Bergmann A, Müller B. Mid-regional pro-adrenomedullin as a prognostic marker in sepsis: an observational study. Crit Care. 2005;9(6):R816-824.

41. Cho SY, Choi JH. Biomarkers of sepsis. Infect Chemother. 2014;46(1):1-12.

42. Paize F, Sarginson R, Makwana N, et al. Changes in the sublingual microcirculation and endothelial adhesion molecules during the course of severe meningococcal disease treated in the paediatric intensive care unit. Intensive Care Med. 2012;38(5):863-871.

43. Fink MP. Cytopathic hypoxia. Is oxygen use impaired in sepsis as a result of an acquired intrinsic derangement in cellular respiration? Crit Care Clin. 2002;18(1):165-175.

44. Jang DH, Greenwood JC, Spyres MB, Eckmann DM. Measurement of Mitochondrial Respiration and Motility in Acute Care: Sepsis, Trauma, and Poisoning. J Intensive Care Med. 2017;32(1):86-94.

45. Harms FA, Bodmer SI, Raat NJ, Mik EG. Non-invasive monitoring of mitochondrial oxygenation and respiration in critical illness using a novel technique. Crit Care. 2015;19:343.

46. Singer M, Deutschman CS, Seymour CW, et al. The Third International Consensus Definitions for Sepsis and Septic Shock (Sepsis-3). JAMA. 2016;315(8):801-810

47. Dellinger RP, Carlet JM, Masur H, et al. Surviving Sepsis Campaign guidelines for management of severe sepsis and septic shock. Crit Care Med. 2004;32(3):858-873.

48. van Genderen ME, Engels N, van der Valk RJ, et al. Early peripheral perfusion-guided fluid therapy in patients with septic shock. Am J Respir Crit Care Med. 2015;191(4):477-480.

49. Monnet X, Marik PE, Teboul JL. Prediction of fluid responsiveness: an update. Ann Intensive Care. 2016;6(1):111.

50. Monnet X, Teboul JL. Volume responsiveness. Curr Opin Crit Care. 2007;13(5):549-553.

51. Monnet X, Teboul JL. Assessment of fluid responsiveness: recent advances. Curr Opin Crit Care. 2018;24(3):190-195.

52. Bortolotti P, Colling D, Colas V, et al. Respiratory changes of the inferior vena cava diameter predict fluid responsiveness in spontaneously breathing patients with cardiac arrhythmias. Ann Intensive Care. 2018;8(1):79.

53. Dare AJ, Phillips AR, Hickey AJ, et al. A systematic review of experimental treatments for mitochondrial dysfunction in sepsis and multiple organ dysfunction syndrome. Free Radic Biol Med. 2009;47(11):1517-1525.

54. Chuang CC, Shiesh SC, Chi CH, et al. Serum total antioxidant capacity reflects severity of illness in patients with severe sepsis. Crit Care. 2006;10(1):R36

55. Rinaldi S, Landucci F, de Gaudio AR. Antioxidant therapy in critically septic patients. Curr Drug Targets. 2009;10(9):872-880.
Open Access Emergency Medicine

\section{Publish your work in this journal}

The Open Access Emergency Medicine is an international, peerreviewed, open access journal publishing original research, reports, editorials, reviews and commentaries on all aspects of emergency medicine. The manuscript management system is completely online and includes a very quick and fair peer-review system, which is all
Dovepress

easy to use. Visit http://www.dovepress.com/testimonials.php to read real quotes from published authors. 